\title{
Response of NiTi SMA wire electrically heated
}

\author{
C. Zanotti ${ }^{\text {a }}$ P. Giuliani, A. Tuissi ${ }^{1}$, S. Arnaboldi ${ }^{1}$, R. Casati $^{1}$ \\ Institute for Energetics and Interphases, Via R. Cozzi, 53 Milano (Italy) \\ ${ }^{1}$ Institute for Energetics and Interphases, Corso Promessi Sposi, 29 Lecco (Italy)
}

\begin{abstract}
In the present work an experimental-numerical approach is used to study the thermo-mechanical behaviour of NiTi wire for defining what parameters are the most important in actuator designing. Tests were carried out heating, by an electrical current, a wire having a diameter of $150 \mu \mathrm{m}$, under constant stresses of $200 \mathrm{MPa}$. Data concerning strain, applied current and voltage are acquired during the tests by PC while wire temperature is recorded by thermographic system.

The numerical code integrates the ordinary differential equation describing the volumetric wire heating and cooling transients assuming that the electrical power is the only external heating source. Temperature distribution in the wire is considered uniform and its change in time depends on wire specific heat, latent heat of the Martensitic-Austenitic transformation, free convection heat exchange coefficient and total emissivity of the surface wire.

Comparisons between experimental and numerical results, obtained under different operating conditions, indicate that the wire heating rate mainly depends on the applied electrical power, latent heat of the Martensitic-Austenitic transformation, free convection heat exchange coefficient.

In the case of the cooling transient, the capability to decrease the wire temperature to reach the ambient value is strongly effected, once the free convection heat exchange coefficient is defined, by the shape of the curve representing the Austenitic-Martensitic transformation.

Simulations of the wire behaviour point out that the time necessary to obtain the maximum strain can be reduce to few $\mathrm{ms}$ if current of $10 \mathrm{~A}$ is used. Moreover, the strain dependence on time can be tailored by choosing the suitable heating transient.
\end{abstract}

\section{Introduction}

The unique functional properties, which are due to a solid state Martensitic transformation, allow nickel titanium shape memory alloys (SMAs) to recover large strains, either spontaneously (Pseudoelasticity PE) or through an increase in temperature (shape memory effect SME) [1].

The SME, exhibited by the Ti rich NiTi alloy, is successfully used in several engineered devices for automotive, appliance, hydraulic, gadgets and biomedical fields [2, 3]. Moreover it has been demonstrated that NiTi wires are suitable for Shape Memory (SM) electrically heated actuators because of their large recoverable strain values (4\%), also under an applied load, with excellent cycling stability (over 100.000 thermo-mechanical cycle) [4]. As intermetallic compound, the NITi shows a rather good workability so it can be drawn down to wires of the order of $100 \mu \mathrm{m}$ (or less) for the realization of promising mini and micro SM actuators. However the functional properties of NiTi wires strongly depend from both thermomechanical manufacturing history and operating conditions.

It this work an experimental equipment, designed and realized for thermo-mechanical cycling of 20-200 $\mu \mathrm{m}$ wire for SM actuators, was used for studying the properties of a commercial wire with $150 \square \mu \mathrm{m}$ in diameter under different applied constant stresses. DSC and Strain/Temperature hysteresis tests of the wire were also carried out in order to have a spread experimental database for the optimization and validation of a numerical model, which is developed for simulating the behavior of the SM wire for actuator.

\footnotetext{
a e-mail: zanotti@ieni.cnr.it
}

This is an Open Access article distributed under the terms of the Creative Commons Attribution-Noncommercial License (http://creativecommons.org/licenses/by-nc/3.0/), which permits unrestricted use, distribution, and reproduction in any noncommercial medium, provided the original work is properly cited. 


\section{Modeling of the wire heating and cooling transients}

The model, describing the volumetric sample heating and cooling transient of NiTi wires heated by electrical power, was developed. Computations are based on the following assumptions:

- electrical power is the only external heating source,

- the temperature distribution in the sample is uniform,

- $\quad$ sample heat capacity is dependent on temperature,

- phase transition is included,

- $\quad$ heat losses by free convection and radiant emission are accounted.

- $\quad$ work done by the wire is considered

The ordinary differential equation describing the wire heating and cooling phenomena can be written as:

$m C_{t}(T) \frac{d T}{d t}=\left(\delta_{1} P_{e l}(T)\right)-\left(P_{c}(T)+P(T)_{e}+\delta_{1} P_{w}(T)\right)$

with the initial boundary condition $T(t=0)=T_{a}$. In particular terms of equation 1 are defined as:

$P_{e l}(T)=\rho L_{w} I^{\wedge} 2 / A_{w}$

the electrical power absorbed by the wire,

$P_{c}(T)=h A_{l}\left(T-T_{a}\right)$

the power loss by free convection,

$P_{w}(T)=w v$

the power necessary to pull up the load,

$P_{e}(T)=\sigma \varepsilon_{T} A_{l}\left(T^{4}-T_{a}^{4}\right)$

the power loss by radiation.

$\delta_{1}$ switch is used to select the contribution of processes during the heating $\left(\delta_{1}=1\right)$ and cooling $\left(\delta_{1}=0\right)$ transients.

$h=N_{u}(T) K_{g} / L_{i}$

$L_{w}=L_{i}-\varepsilon$

$A_{w}=V_{w} / L_{w}$

$A_{l}=A_{w} L_{w}$

$\rho=\left(V A_{w}\right) /\left(I L_{w}\right)$.

$A_{l} \quad$ lateral area of the wire

$A_{w} \quad$ wire cross section ares

$C_{t} \quad$ total specific heat

$h$ free convection coefficient

I current

$K_{g} \quad$ gas thermal conductivity

$L_{i} \quad$ wire initial length

$L_{w} \quad$ wire length

$\begin{array}{ll}t_{h} & \text { heating time } \\ T & \text { wire temperature }\end{array}$

$T_{a} \quad$ ambient temperature

$\mathrm{V} \quad$ voltage

v deformation velocity

$V_{w} \quad$ wire volume

$m \quad$ wire mass

$\mathrm{W}$

load

$\varepsilon_{T} \quad$ wire total emissivity

$N_{u} \quad$ Nusselt number

wire resistivity

$t \quad$ time

$\rho$

$\varepsilon \quad$ wire strain

In order to integrate the first order differential equation (1) $C_{t}(T), h(T), \rho(T), L_{w}(T), A_{w}(T), v(T)$ and $\varepsilon_{T}$ must be chosen and a fourth order Runge-Kutta method has been used. 


\section{Experimental apparatus and procedure}

To evaluate the cycling behavior of SMA wires in a range of 20-200 $\mu \mathrm{m}$ in diameter, as well as the fatigue resistance of the material and its functional characteristics, a self-assembly system was designed and realized (Fig1). The wire was vertically arranged and was constrained to the system structure through an upper clamp and to induce a constant stress into the material, an axial weight was coupled to a lower vertical sliding block, directly hanged by the NiTi wire (Fig 2).

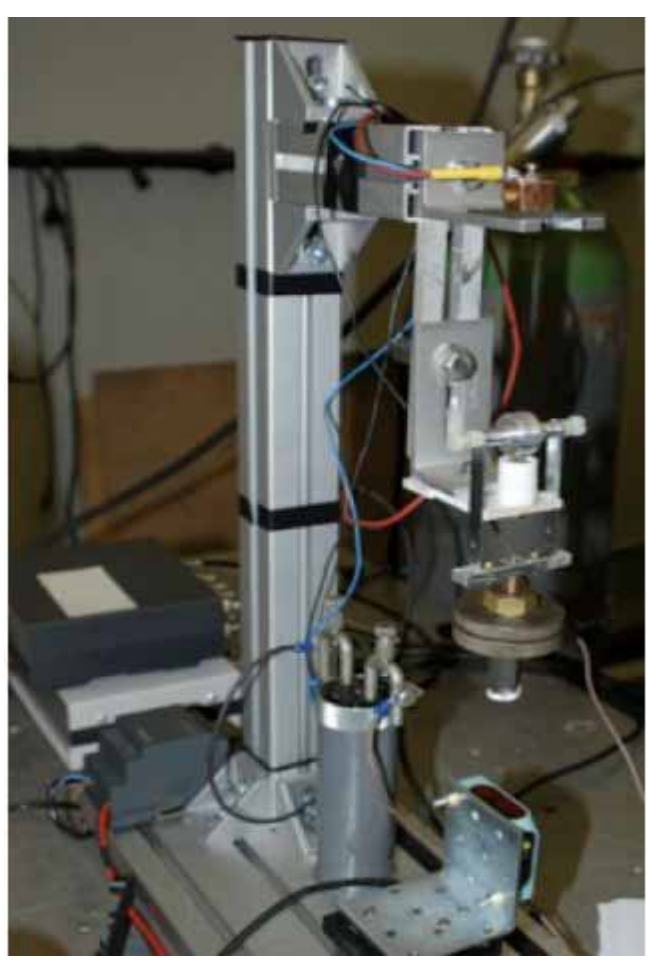

Fig. 1. Self-Assembled Cycling Machine

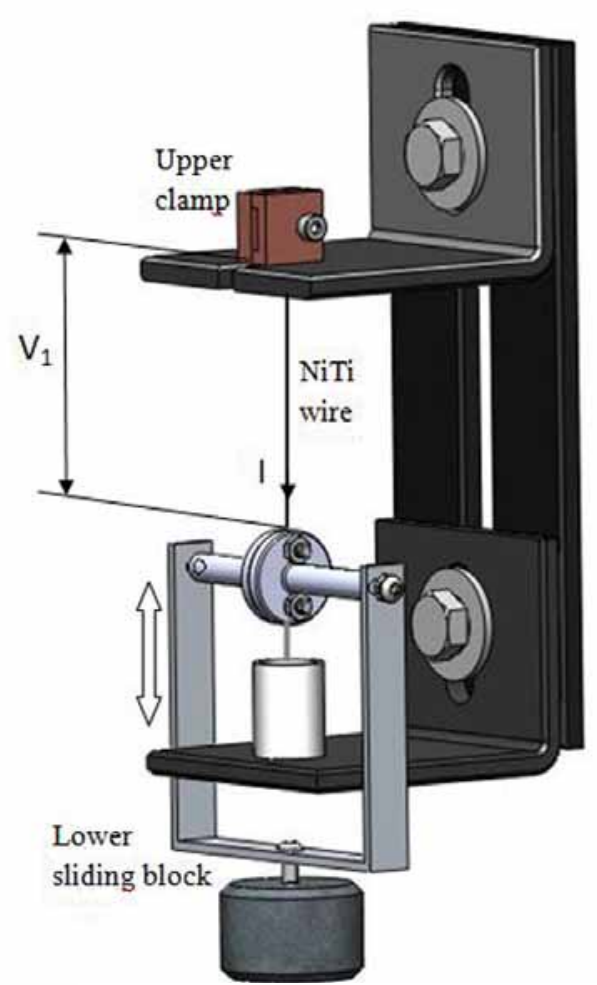

Fig. 2. Wire clamping system

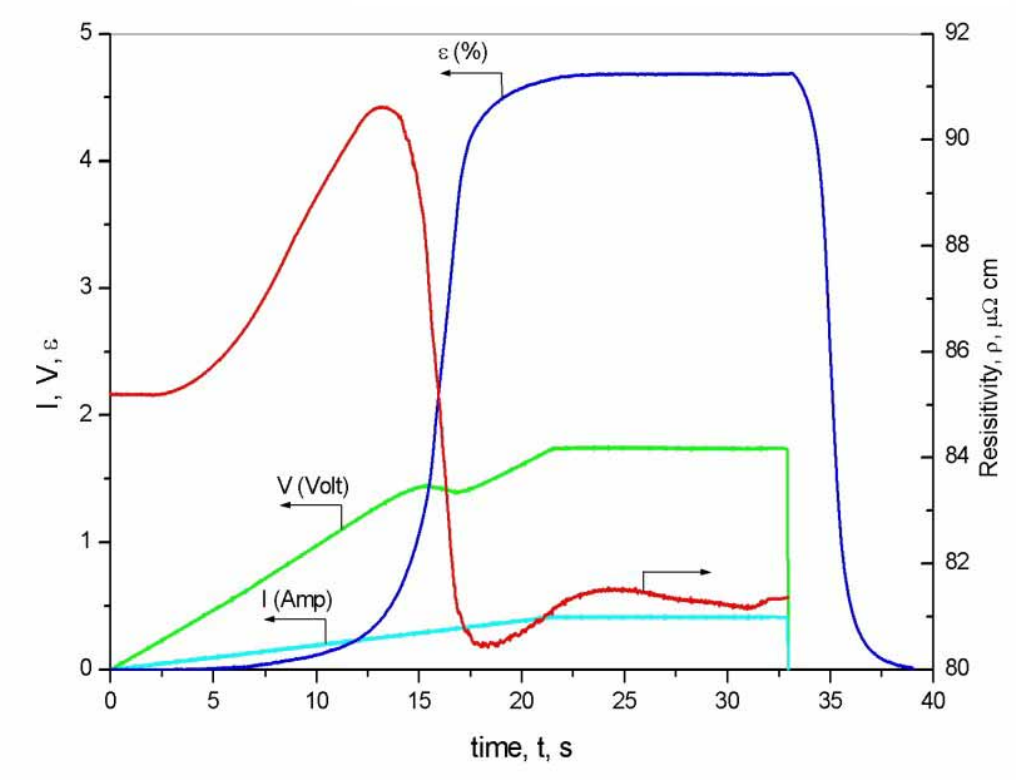

Fig. 3. Experimental data and calculated resistivity

To avoid constraints that can generate anomalous stress during the wire shortening, a mercury bath was used to guarantee the electrical contact of the movable wire side.

The electrical circuit allows to supplying the current in different ways and increasing rates controlling the current source by a PC.

The clamps, made of conductor metals, were directly joined to an electric current generator.

The wire was heated up by Joule effect and the wire deformation, during the thermo mechanical cycling, was detected by a laser optical proximity sensor.

Data concerning strain, applied current and voltage were acquired during the tests by a second PC while, the wire temperature was recorded by a thermal video system Hughes series 3000. 


\section{Experimental and numerical results}

The methodology is based on the capability to define, by experimental tests, suitable data necessary for computing the wire heating and cooling transients. For this reason, tests, aimed to determine the wire electrical resistivity, are carried out under constant stress and typical results are reported in Fig. 3. Here, the acquired current, voltage and strain, relevant the wire (diameter $=150 \mu \mathrm{m}, L_{i}=100 \mathrm{~mm}$ ) under a stress of $200 \mathrm{MPa}$ and heated by peculiar current time dependence are plotted. In this case, the wire heating time is long enough to evidence the effect of the phenomena occurring during the heating transient.

First of all, by these data, $L_{w}(t), A_{w}(t)$ can be calculated while $v(t)$ is obtained by first derivation of the $L_{w}(t)$. Then, the electrical resistivity dependence on time can be estimated by eq. 10 and it is reported in Fig. 3 too. The shape of this curve shows a decreases in the time range where the strain increases indicating the effect of the MartensiticAustenitic transformation on this parameter.

Once all these terms and the temperature data, recorded by the infrared thermocamera, are known this test can be simulated till to overlap the computed wire temperature on the experimental one as shown in Fig.4. Moreover, $\varepsilon(T)$ and $C_{t}(T)$ can be defined and their dependence on temperature shown in Fig. 5. Many experimental tests relevant to the wire having a stress of $200 \mathrm{MPa}$ and featured by different current histories were processed to get the data necessary for simulations.

Once, $\varepsilon(T), N_{u}(T), K_{g}(T), C_{t}(T)$ and $\varepsilon$ are known for each operating condition (stress $200 \mathrm{MPa}$ ), equation 1 can be integrated and different heating transient computed to find out the wire behaviour.

In particular, $C_{t}$, was modelled starting from DSC data and results found in literature $[6,7]$ and a gaussian dependence on temperature has been chosen [8].

$N_{u}(T)$ has been designed on the basis of data reported in $[9,10]$ and the total wire emissivity $\varepsilon$ was considered constant. Values of $K_{g}(T)$ can be found in [11] and of $\varepsilon$ in [12].

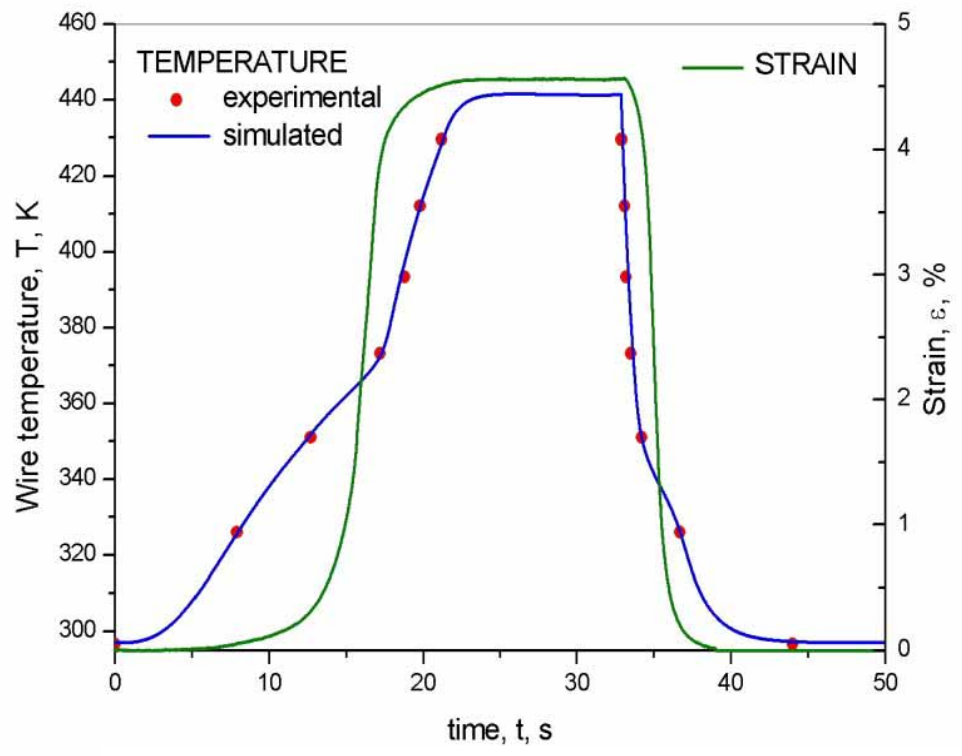

Fig. 4. Wire temperature: experimental and numerical results

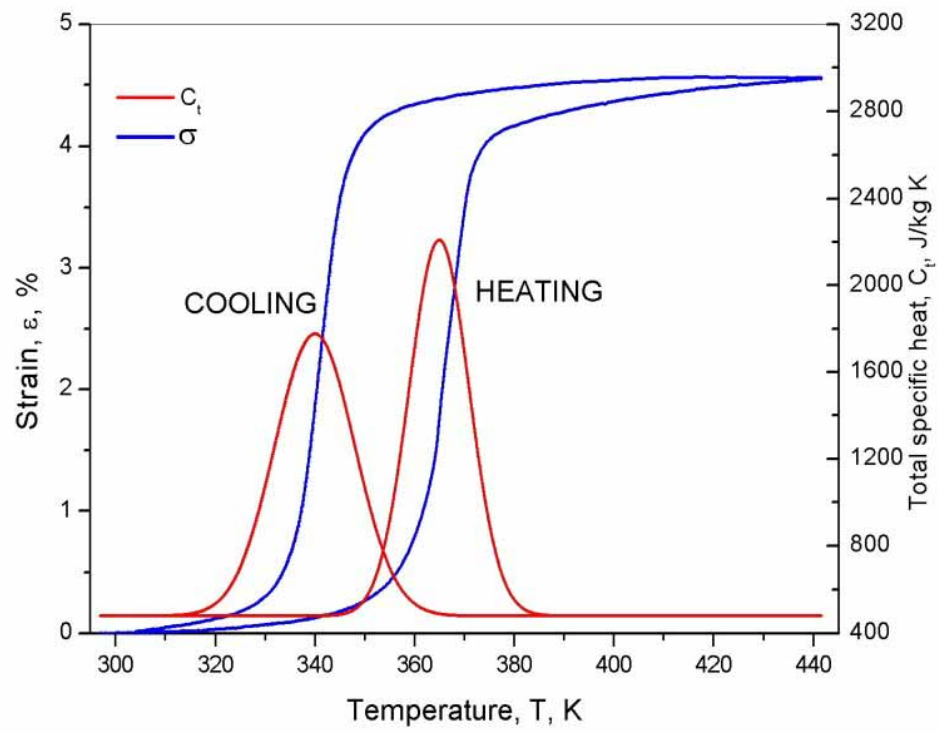

Fig. 5. Total scpecific heat and strain features 


\section{Simulated wire behaviour}

Simulations are computed using the data obtained testing the $150 \mu \mathrm{m}$ wire under constant stress of $200 \mathrm{MPa}$.

First of all, it is interesting to indicate which is the influence of $C_{t}$ on the wire strain and temperature dependence on time.

In Fig. 6 curves regarding $\varepsilon$ (red and magenta lines) and $\mathrm{T}$ (black and blue lines) obtained driven the wire heating by a constant current of $0.45 \mathrm{~A}$ are reported.

Here the red and black lines are computed using a constant value of $C_{t}$ $(480 \mathrm{KJ} / \mathrm{Kg})$ while the magenta and blue lines are obtained by a gaussian dependence of $C_{t}$ on temperature.

It is evident that the MartensiticAustenitic transformation induces a delay in reaching the maximum value of $\mathrm{T}$ and $\varepsilon$ and this occurrence is due to the heat absorbed during the transformation as indicated by the DSC data.

The same effect, now due to the heat released, is observed during the cooling transient in the temperature range where the reverse Austenitic-Martensitic transformation occurs. This aspect underlines that the time to complete a cycle is increases.

Other cases were analyzed to point out the influence of the current (square wave form) on the time to reach the maximum strain $\left(t_{h}\right)$. All these simulations were obtained reaching the maximum wire temperature of $450 \mathrm{~K}$.

Results are reported in Fig. 7, where different curves, representing the strain dependence on time computed using five current values, show that $t_{h}$ decreases with the current increase.

Of course, the cooling transient is always described by the same curve.

These kind of simulations have been computed ranging the current from 0.43 to $10 \mathrm{~A}$ and data relevant the dependence on the electrical current $t_{h}$ are depicted in Fig. 8.

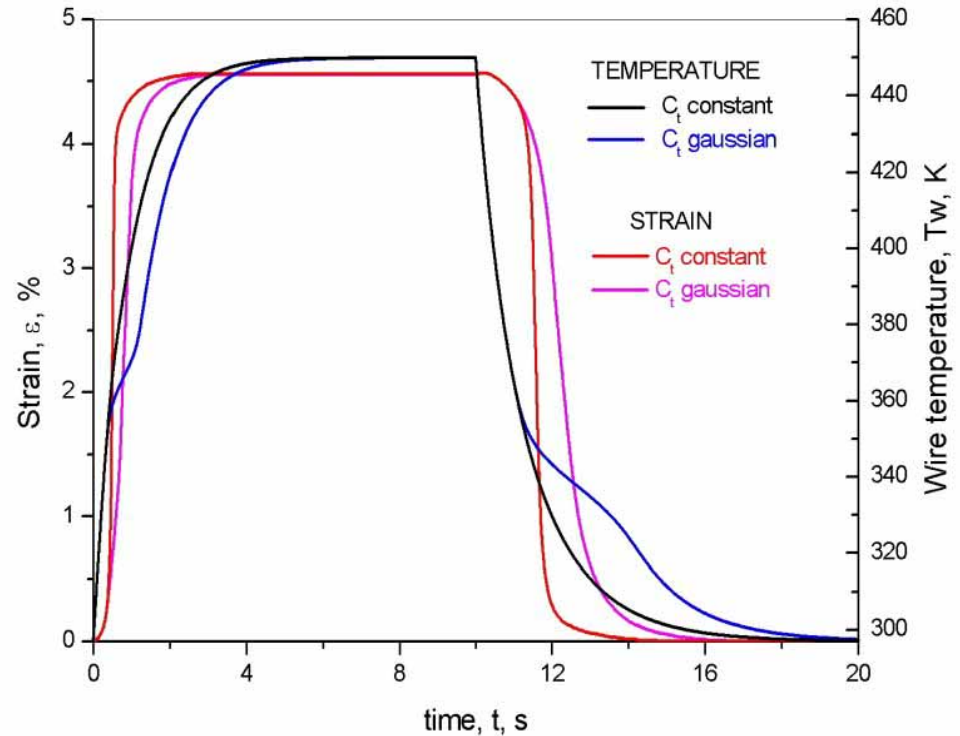

Fig. 6. Effect of $C_{t}$ on strain and temperature

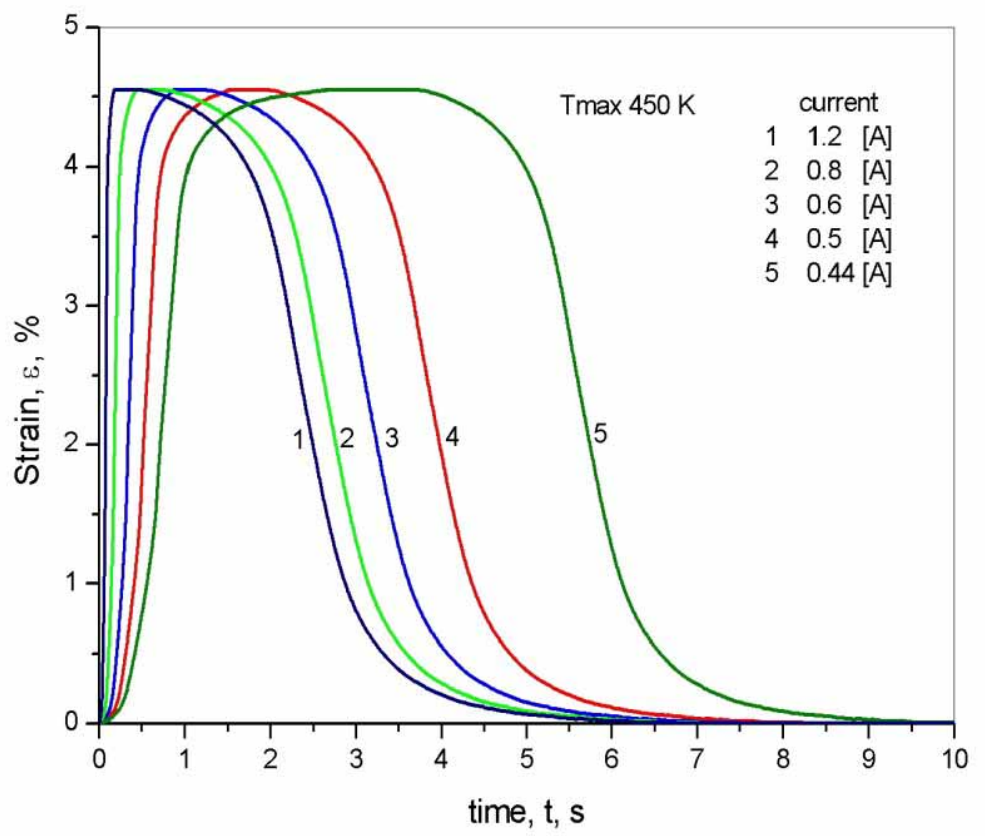

Fig. 7. Influence of the heating current on strain 
These results show the strong decreases of the $t_{h}$ when the current reaches $10 \mathrm{~A}$ and indicate that, from the thermal point of view, the wire deformation can occurs in very a short time $(0.0025 \mathrm{~s})$.

This aspect can be acceptable supposing that the M-A transformation time, if any, is considered to be close to 0 .

Moreover, in Fig. 8 data concerning the electrical energy absorbed during the wire heating transient necessary to reach the complete M-A transformation are reported. The trend of these data shows that the electrical energy reaches low values when the current is larger than $3 \mathrm{~A}$.

This can be explained considering that when current is $3 \mathrm{~A}$ the heating time is $0.028 \mathrm{~s}$.

Under this condition the heat loss by free convection is reduced thus, almost all the electrical power is absorbed by the wire.

Finally, different ways to increase the current, till to reach the maximum temperature of $440 \mathrm{~K}$, can be used to simulate the wire behaviour, as shown in Fig. 9.

The influence of the current history on the wire heating transient is depicted in Fig. 10 that reports the relevant strains and wire temperatures.

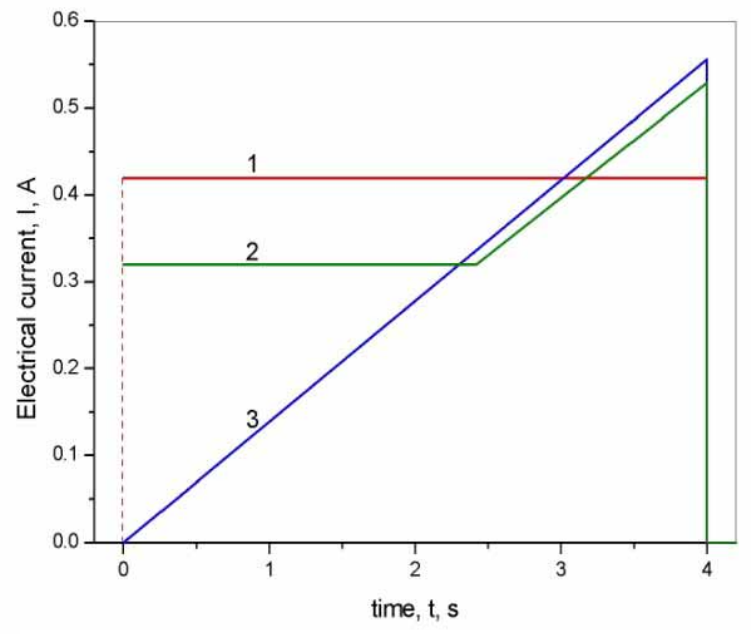

Fig. 9. Different electrical current history

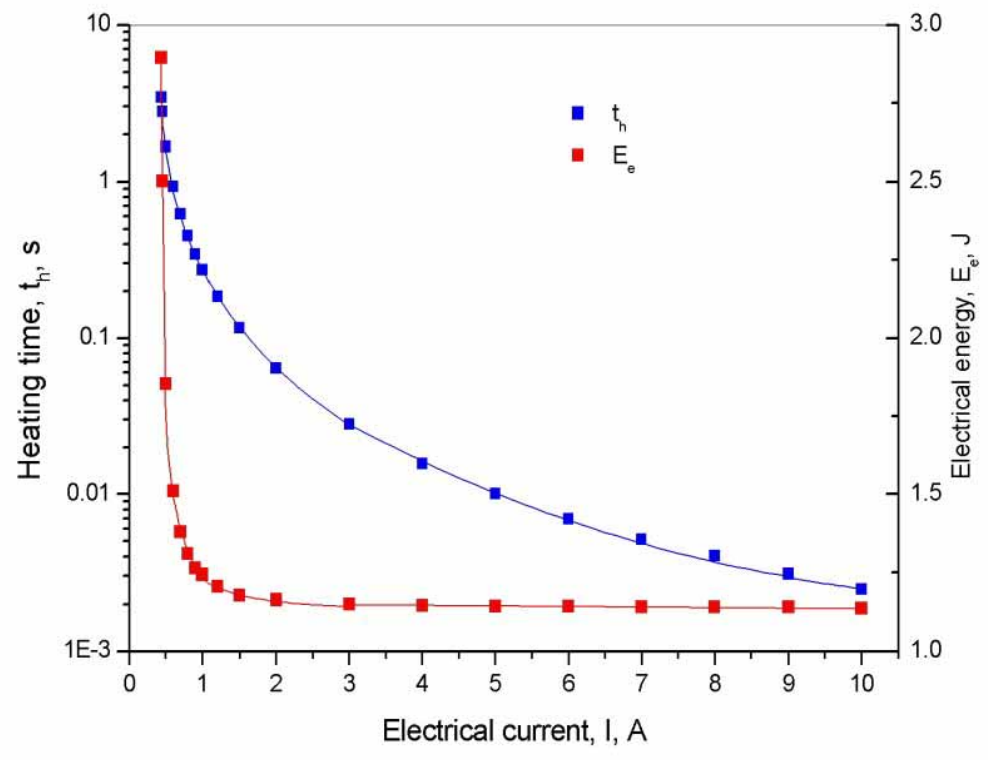

Fig. 8. Dependence of heating time and absorbed energy on current

These results point out that the wire length can be shortened in the same time but following different histories featured by fast deformation at the beginning (curve 1) or fast deformation at the end (curve 3 ) of the heating transient.

This possibility depends on the capability, once the $C_{t}$ and operating condition $(h)$ are defined, to properly change the wire temperature increase during the M-A transformation.

In particular, the square wave current (1) gives the faster wire heating and the wire is shortened at the beginning of the heating transient.

On the contrary, when current is linearly increased (3) the opposite behaviour occurs. Of course, a mixture of the case 1 and 3 gives an intermediate result (2). 


\section{Conclusions}

An experimental-numerical approach useful to analyze the thermo-mechanical behaviour of NiTi SMAs wire has been presented. Wire, featured by a diameter of $150 \mu \mathrm{m}$, has been experimentally tested under constant stress of $200 \mathrm{MPa}$.

Experimental tests were carried out heating the wire by electrical power and current, voltage, strain and wire temperature were acquired simultaneously.

These data were used to simulate the experimental tests for estimating the dependence on the wire temperature of the electrical resistivity, the Nusselt number driving the heat exchange by free convection and the total specific heat of the NiTi wire.

Finally, on the basis of the obtained data describing the wire features when a constant stress of $200 \mathrm{MPa}$ is applied, numerical simulations have been performed to define the wire behaviour during the heating and cooling transient.

Computed results indicate that the heating time, necessary to complete the M-A transformation can be reduced to few ms heating the wire by a current of 10 A while the cooling time can not be changed without modifying the operating conditions. Moreover, short heating times require less electrical energy to complete the M-A transformation.

Finally, the wire length history can be tailored choosing the suitable current dependence on time able to properly increase the wire temperature.

\section{References}

[1] Shape Memory Materials; Ed. by K.Otsuka and C.M.Wayman - Cambridge univ. Press 1998.

[2] J.Van Humbeeck, "Non-medical applications of shape memory alloys", Materials Science and Engineering A, Volume: 273-275, (1999), pp. $134-148$.

[3] T. Duerig, A. Pelton, D. Stöckel "An overview of nitinol medical applications", MaterialsScience and Engineering A, Volume: 273-275, (1999), pp. 149 - 160.

[4] A. Tuissi, P. Bassani, A. Mangioni, L. Toia, F. Butera, "Fabrication process and characterization of NiTi wires for actuators", Proceedings of SMST 2004 ed. M.Mertmann (2006) pp 501-508.

[5] V.Antonucci, G. Faiella, M. Giordano, F. Mennella, L. Nicolais, "Electrical resistivity study and characterization during NiTi phase transformations" Thermochimica Acta 462 (2007) 64-69.

[6] J.F. Smith "The heat capacity of solid Ni-Ti alloys in the temperature range 120 to $800 \mathrm{~K}$ " Journal of phase equilibria vol 14, No 4, (1993).

[7] M. Rhode, A.Schussler, "On the responce-time behaviour of laser micromachined NiTi shape memory actuators" Sensor and actuators A61 (1997) 463-468.

[8] C. Zanotti, P. Giuliani P. Bassani, Z. Zhang and A. Chrysanthou, "Comparison between the thermal properties of fully dense and porous Ni-Ti SMAs", Intermetallics, doi:10.1016/j.internet.2009.06.001.

[9] Bhattacharyya, L. Sweeney, M.G. Faulkner "Experimental characterization of free convection during thermal phase transformations in shape memory alloy wires" Smart Mater. Struct. 11 (2002) 411-422.

[10] C.O. Popiel, J. Wojtkowiak, K. Bober "Laminar free convective heat transfer from isothermal vertical slender cylinder" Experimental Thermal and Fluid Science 32 (2007) 607-613.

[11] F.P. Incropera D.P. De Witt "Fundamentals of heat and mass transfer" ed. Wiley.

[12] "Handbook and Enciclopedia Temperature", volume MM, 2000, Omega. 